\title{
SCATTERED COMPACT SETS IN CONTINUOUS IMAGES OF ČECH-COMPLETE SPACES
}

\author{
TARAS BANAKH, BOGDAN BOKALO, VLADIMIR TKACHUK \\ Dedicated to the 80-th birthday of A.V. Arhangel'skii
}

\begin{abstract}
Assume that a functionally Hausdorff space $X$ is a continuous image of a Čech complete space $P$ such that $l(P)<\mathfrak{c}$. Then the following conditions are equivalent: (i) every compact subset of $X$ is scattered, (ii) for every continuous map $f: X \rightarrow Y$ to a functionally Hausdorff space $Y$ the image $f(X)$ has cardinality not exceeding $\max \{l(P), \psi(Y)\}$, (iii) no continuous map $f: X \rightarrow[0,1]$ is surjective. We also prove the equivalence of the conditions: (a) $\omega_{1}<\mathfrak{b}$, and (b) a K-analytic space $X$ (with a unique non-isolated point) is countable iff every compact subset of $X$ is countable.
\end{abstract}

A topological space $X$ is scattered if every non-empty subspace of $X$ contains an isolated point.

Definition 1. A topological space $X$ is $k$-scattered if every compact subspace of $X$ is scattered.

By [6, p. 34], a Cech-complete space is $k$-scattered if and only if it is scattered. We recall that a Tychonoff space $X$ is $\breve{C}$ ech-complete if $X$ is a $G_{\delta}$-set in some (equivalently, any) compactification of $X$, see [8, 3.9.1]. A topological space is Polish if it is homeomorphic to a separable complete metric space. It is well-known [8, 4.3.26] that each Polish space is Čech-complete.

The following well-known characterization of scattered compact Hausdorff spaces can be found in [1, §3] or [14, 8.5.4].

Theorem 1. For a compact Hausdorff space $X$ the following conditions are equivalent:

(1) $X$ is scattered;

(2) for any continuous map $f: X \rightarrow Y$ to a metrizable space $Y$ the image $f(X)$ is at most countable;

(3) no continuous map $f: X \rightarrow[0,1]$ is surjective.

In this paper we generalize this characterization to K-analytic spaces, more generally, to functionally Hausdorff spaces $X$ with analyticity number $\alpha(X)<\mathfrak{c}$. Here $\mathfrak{c}$ stands for the cardinality of the continuum.

We recall that a topological space $X$ is functionally Hausdorff if for any distinct points $x, y \in X$ there exists a continuous map $f: X \rightarrow[0,1]$ such that $f(x)=0$ and $f(y)=1$.

Next, we recall the definitions of some cardinal topological invariants.

The Lindelöf number $l(X)$ of a topological space $X$ is a smallest infinite cardinal $\kappa$ such that any open cover $\mathcal{U}$ of $X$ has a subcover $\mathcal{V} \subset \mathcal{U}$ of cardinality not exceeding $\kappa$. A topological space $X$ is called Lindelö if $l(X) \leq \omega$.

The pseudocharacter $\psi(X)$ of a topological space $X$ is the smallest cardinal $\kappa$ such that for any point $x \in X$ the family $\tau_{x}$ of all open neighborhoods of $x$ contains a subfamily $\mathcal{U}_{x} \subset \tau_{x}$ of cardinality $\left|\mathcal{U}_{x}\right| \leq \kappa$ such that $\bigcap \mathcal{U}_{x}=\bigcap \tau_{x}$.

A topological space $X$ is defined to be

- analytic if $X$ is a continuous image of a Polish space;

- $K$-analytic if $X$ is a continuous image of a Lindelöf Čech-complete space;

- $\kappa$-analytic for a cardinal $\kappa$ if $X$ is a continuous image of a Čech-complete space $P$ such that $l(P) \leq \kappa$.

1991 Mathematics Subject Classification. 54H05, 03E15, 03E17.

Key words and phrases. Čech-complete space, scattered space, $k$-scattered space, K-analytic space. 
Analytic and K-analytic spaces play an important role in General Topology [2, [10, Descriptive Set Theory [12, 13. and Functional Analysis [11.

For a topological space $X$ its analyticity $\alpha(X)$ is defined as the smallest cardinal $\kappa$ for which the space $X$ is $\kappa$-analytic. Since every topological space $X$ is a continuous image of a discrete (and hence Cech-complete) space, the analyticity is a well-defined cardinal invariant such that

$$
l(X) \leq \alpha(X) \leq|X|+\omega .
$$

For a Cech-complete space its analyticity is equal to the Lindelöf number. Observe that a topological space $X$ is $K$-analytic if and only if $\alpha(X)=\omega$.

Theorem 2. Let $f: X \rightarrow Y$ be a continuous map from a Čech-complete space $X$ to a functionally Hausdorff space $Y$. If $|f(X)|>\max \{\psi(Y), l(X)\}$, then there exists a compact subset $K \subset X$ whose image $f(K)$ is not scattered.

Theorem 2 will be proved in Section [1. Now we apply this theorem to prove the following characterization.

Theorem 3. For a functionally Hausdorff space $X$ with $\alpha(X)<\mathfrak{c}$, the following conditions are equivalent:

(1) $X$ is k-scattered;

(2) For any continuous map $f: X \rightarrow Y$ to a functionally Hausdorff space $Y$, the image $f(X)$ has cardinality not exceeding $\max \{\alpha(X), \psi(Y)\}$.

(3) For any continuous map $f: X \rightarrow Y$ to a metrizable space $Y$, the image $f(X)$ has cardinality strictly less than $\mathfrak{c}$.

(4) No continuous map $f: X \rightarrow[0,1]$ is surjective.

Proof. $(1) \Rightarrow(2)$ Assume that every compact subspace of $X$ is scattered. To derive a contradiction, assume that for some continuous map $f: X \rightarrow Y$ to a functionally Hausdorff space $Y$, the image $f(X)$ has $|f(X)|>\max \{\alpha(X), \psi(Y)\}$. By the definition of the cardinal $\alpha(X)$, there exists a continuous surjective map $g: P \rightarrow X$ from a Čech-complete space $P$ such that $l(P)=\alpha(X)$. Since $|f \circ g(P)|=|f(X)|>\max \{\alpha(X), \psi(Y)\}=\max \{l(P), \psi(Y)\}$, we can apply Lemma 2] and find a compact subset $K \subset P$ whose image $f \circ g(K)$ is not scattered. Then the compact subset $g(K)$ of $X$ is not scattered, too.

The implications $(2) \Rightarrow(3) \Rightarrow(4)$ are trivial.

$(4) \Rightarrow(1)$ Assume that $X$ contains a non-scattered compact subset $K \subset X$. By Theorem 1 . there exists a continuous surjective map $f: K \rightarrow[0,1]$. The space $X$, being functionally Hausdorff, admits a continuous injective map $g: X \rightarrow Y$ to a compact Hausdorff space $Y$. By the compactness of $K$, the restriction $g\lceil K: K \rightarrow Y$ is a closed topological embedding. By the Tietze-Urysohn Theorem [8, 2.1.8], the continuous map $f \circ\left(g\lceil K)^{-1}: g(K) \rightarrow[0,1]\right.$ has a continuous extension $\varphi: Y \rightarrow[0,1]$. Then $\bar{f}=\varphi \circ g: X \rightarrow[0,1]$ is a continuous extension of the map $f$. The surjectivity of $f$ implies the surjectivity of $\bar{f}$.

Theorem 3 has the following corollary extending the classical result of Souslin on cardinality of analytic spaces, see [12, 14.13].

Corollary 1. Any functionally Hausdorff space $X$ has cardinality

$$
|X| \leq \max \{\alpha(X), \psi(X)\} \text { or }|X| \geq \mathfrak{c} .
$$

In particular, any analytic functionally Hausdorff space $X$ has cardinality $|X| \in \omega \cup\{\omega, \mathfrak{c}\}$.

For $F_{\sigma \delta}$-subsets of compact Hausdorff spaces, the following corollary of Theorem 3 is known, see, e.g., [15, Problem 262].

Corollary 2. A functionally Hausdorff $K$-analytic space $X$ is at most countable if and only if $X$ has countable pseudocharacter and every compact subset of $X$ is at most countable. 
Looking at Corollary 2 it is natural to ask 3 whether the countability of the pseudocharacter can be removed from this characterization. It turns out that this can be done if and only if $\omega_{1}<\mathfrak{b}$. We recall [7, [16], [5] that $\mathfrak{b}$ is the smallest cardinality of a subset $B \subset \omega^{\omega}$ such that for every $y \in \omega^{\omega}$ there exists $x \in B$ such that $x \mathbb{Z}^{*} y$, where $x \leq^{*} y$ means that the set $\{n \in \omega: x(n) \not \leq y(n)\}$ is finite. It is well-known [7, [16], [5] that $\omega_{1}<\mathfrak{b}=\mathfrak{c}$ under MA $+\neg \mathrm{CH}$. On the other hand, $\omega_{1}=\mathfrak{b}=\mathfrak{c}$ under $\mathrm{CH}$.

The equivalence $(1) \Leftrightarrow(2)$ in the following theorem was proved by Fremlin [9].

Theorem 4. The following conditions are equivalent:

(1) $\omega_{1}<\mathfrak{b}$.

(2) A K-analytic Hausdorff space $X$ is analytic iff every compact subset of $X$ is metrizable.

(3) A K-analytic space $X$ is countable iff every compact subset of $X$ is countable.

(4) A K-analytic Hausdorff space $X$ with a unique non-isolated point is countable iff every compact subset of $X$ is countable.

Proof. Since $(1) \Leftrightarrow(2)$ was established by Fremlin [9], it remains to prove that $(1) \Rightarrow(3) \Rightarrow(4) \Rightarrow$ (1). The implication $(3) \Rightarrow(4)$ is trivial.

$(1) \Rightarrow(3)$. Assume that $\omega_{1}<\mathfrak{b}$ and take any $\mathrm{K}$-analytic space $X$ such that every compact subset of $X$ is countable. By definition, the $\mathrm{K}$-analytic space $X$ is the image of a Lindelöf Čech-complete space $P$ under a continuous surjective map $f: P \rightarrow X$. Let $\bar{P}$ be a compactification of $P$ and let $\left(W_{n}\right)_{n \in \omega}$ be a decreasing sequence of open sets in $\bar{P}$ such that $P=\bigcap_{n \in \omega} W_{n}$. Since $X$ is Lindelöf, we can assume that each open set $W_{n}$ is $\sigma$-compact. So, $W_{n}=\bigcup_{m \in \omega} K_{n, m}$ for an increasing sequence $\left(K_{n, m}\right)_{m \in \omega}$ of compact subsets of $\bar{P}$. For every infinite sequence $s \in \omega^{\omega}$ consider the compact set $K_{s}=\bigcap_{n \in \omega} K_{n, s \uparrow n} \subset \bigcap_{n \in \omega} W_{n}=P$ and observe that $X=f(P)=\bigcup_{s \in \omega^{\omega}} f\left(K_{s}\right)$, and $K_{s} \subset K_{t}$ for any sequences $s \leq t$ in $\omega^{\omega}$. By our assumption, all compact sets in $X$ are at most countable. Consequently, for every $s \in \omega^{\omega}$ the compact set $f\left(K_{s}\right)$ is at most countable.

Assuming that $X$ is uncountable, we can construct transfinite sequences of points $\left\{s_{\alpha}\right\}_{\alpha<\omega_{1}} \subset$ $\omega^{\omega}$ and $\left\{x_{\alpha}\right\}_{\alpha \in \omega^{\omega}} \subset X$ such that $x_{\alpha} \in f\left(K_{s_{\alpha}}\right) \backslash \bigcup_{\beta<\alpha} f\left(K_{s_{\beta}}\right)$ for every $\alpha<\omega_{1}$.

The definition of the cardinal $\mathfrak{b}>\omega_{1}$ implies that there exists a countable subset $T \subset \omega^{\omega}$ such that for any $\alpha<\omega_{1}$ there exists $t \in T$ with $s_{\alpha} \leq t$. By the Pigeonhole Principle, for some $t \in T$ the set $\Omega=\left\{\alpha<\omega_{1}: s_{\alpha} \leq t\right\}$ is uncountable. Then the countable set $f\left(K_{t}\right)$ contains the uncountable set $\bigcup_{\alpha \in \Omega} f\left(K_{x_{\alpha}}\right) \supset\left\{x_{\alpha}: \alpha \in \Omega\right\}$, which is a desired contradiction, showing that $X$ is at most countable.

(4) $\Rightarrow(1)$ Assuming that $\omega_{1}=\mathfrak{b}$, we can find a transfinite sequence $\left\{x_{\alpha}\right\}_{\alpha \in \omega_{1}} \subset \omega^{\omega}$ such that for every $y \in \omega^{\omega}$ there exists $\alpha \in \omega_{1}$ such that $x_{\alpha} \mathbb{Z}^{*} y$. Using the definition of $\mathfrak{b}$, for every $\alpha<\omega_{1}$ choose a function $y_{\alpha} \in \omega^{\omega}$ such that $x_{\alpha} \leq y_{\alpha}$ and $x_{\beta} \leq y_{\beta} \leq^{*} y_{\alpha}$ for all $\beta<\alpha$. Then for every $y \in \omega^{\omega}$ the set $\left\{\alpha \in \omega_{1}: y_{\alpha} \leq y\right\}$ is countable, which implies that for every compact subset $K \subset \omega^{\omega}$ the intersection $K \cap\left\{y_{\alpha}\right\}_{\alpha \in \omega_{1}}$ is countable.

Let $Y=\left\{y_{\alpha}\right\}_{\alpha \in \omega_{1}}$ and $X=\{\infty\} \cup Y$ where $\infty \notin Y$ is any point. Endow $X$ with the Hausdorff topology generated by the base

$$
\{\{y\}: y \in Y\} \cup\left\{X \backslash D: D \subset Y \text { is closed and discrete in } \omega^{\omega}\right\}
$$

and observe that $\infty$ is a unique non-isolated point of $X$, which implies that the space $X$ is (hereditarily) normal.

Consider the compact-valued function

$$
\Phi: \omega^{\omega} \multimap X, \Phi: x \mapsto\{\infty, x\} \cap X .
$$

and observe that it is upper semi-continuous in the sense that for every open set $U \subset X$ the set $\left\{x \in \omega^{\omega}: \Phi(x) \subset U\right\}$ is open in $\omega^{\omega}$. Since $X=\bigcup_{x \in \omega^{\omega}} \Phi(x)$, the space $X$ is K-analytic, see [10, 3.1].

We claim that every compact subset $K \subset X$ is countable. To derive a contradiction, assume that $K$ is uncountable. Then $K \cap Y$ is uncountable and the closure $\overline{K \cap Y}$ of $K \cap Y$ in $\omega^{\omega}$ is not compact (as the intersection of $Y$ with any compact subset of $\omega^{\omega}$ is countable). Since $\overline{K \cap Y}$ is not compact, there exists an infinite subset $D \subset K \cap Y$, which is closed and discrete in $\omega^{\omega}$. The 
definition of the topology of $X$ ensures that the infinite set $D \subset K$ is closed and discrete in $X$, which is not possible as $K$ is compact.

\section{Proof of Theorem 2}

Let $f: X \rightarrow Y$ be a continuous surjective map from a Čech-complete space $X$ into a functionally Hausdorff space $Y$ and $\kappa=\max \{l(X), \psi(Y)\}$. The space $X$, being Cech-complete, is a $G_{\delta}$-set in some compactification $\bar{X}$ of $X$. Then there exists a decreasing sequence $\left(W_{n}\right)_{n \in \omega}$ of open sets in $\bar{X}$ such that $X=\bigcap_{n \in \omega} W_{n}$ and $W_{0}=\bar{X}$.

Let $2=\{0,1\}$ and $2^{<\omega}=\bigcup_{n \in \omega} 2^{n}$ be the family of all finite binary sequences. For a binary sequence $s=\left(s_{0}, \ldots, s_{n-1}\right) \in 2^{<\omega}$ and a number $i \in\{0,1\}$ by $\hat{s} i$ we denote the sequence $\left(s_{0}, \ldots, s_{n-1}, i\right)$.

Assuming that $|f(X)|>\kappa$, we shall inductively construct a family $\left(U_{s}\right)_{s \in 2<\omega}$ of open $\sigma$-compact sets in $\bar{X}$ such that for every $n \in \omega$ and $s \in 2^{n}$ the following conditions are satisfied:

$\left(a_{s}\right)\left|f\left(X \cap U_{s}\right)\right|>\kappa$

$\left(b_{s}\right) \bar{U}_{s^{\wedge} 0} \cup \bar{U}_{s^{\wedge} 1} \subset U_{s} \subset W_{n}$

$\left(c_{s}\right) \bar{U}_{s \wedge} \cap \bar{U}_{s \wedge 1}=\emptyset$;

$\left(d_{s}\right) f\left(X \cap \bar{U}_{s^{\wedge} 0}\right) \cap f\left(X \cap \bar{U}_{s^{\wedge} 1}\right)=\emptyset$.

We start the inductive construction letting $U_{\emptyset}=\bar{X}$ for the unique element $\emptyset \in 2^{0}$. Assume that for some $n \in \omega$ and $s \in 2^{n}$ we have constructed an open $\sigma$-compact set $U_{s} \subset \bar{X}$ satisfying the condition $\left(a_{s}\right)$. We shall construct two open $\sigma$-compact subsets $U_{s^{\wedge} 0}, U_{s^{\wedge} 1}$ in $U_{s}$ satisfying the conditions $\left(b_{s}\right),\left(c_{s}\right),\left(d_{s}\right)$ and $\left(a_{s^{\wedge} 0}\right),\left(a_{s^{\wedge} 1}\right)$.

Let $Y_{s} \subset Y$ be the set of points $y \in Y$ possessing an open neighborhood $O_{y} \subset Y$ such that the set $O_{y} \cap f\left(X \cap U_{s}\right)$ has cardinality $\leq \kappa$. We claim that the set $f(X) \backslash Y_{s}$ is infinite. To derive a contradiction, assume that the set $f(X) \backslash Y_{s}$ is finite. Since the space $Y$ is Hausdorff with $\psi(Y) \leq \kappa$, there exists a family $\mathcal{V}$ of open sets in $Y$ such that $|\mathcal{V}| \leq \kappa$ and $\bigcap \mathcal{V}=f(X) \backslash Y_{s}$. Observe that for every $V \in \mathcal{V}$, for the closed subspace $f(X) \backslash V$ of the space $f(X)$ we have $l(f(X) \backslash V) \leq l(f(X)) \leq l(X) \leq \kappa$. Then there exists a set $Y_{V} \subset f(X) \backslash V \subset Y_{s} \backslash V$ of cardinality $\left|Y_{V}\right| \leq l(f(X) \backslash V)$ such that $f(X) \backslash V \subset \bigcup_{y \in Y_{V}} O_{y}$. Then

$\kappa<\left|f\left(X \cap U_{s}\right)\right|=\left|f\left(X \cap U_{s}\right) \backslash Y_{s}\right|+\left|f\left(X \cap U_{s}\right) \cap Y_{s}\right| \leq\left|f(X) \backslash Y_{s}\right|+\sum_{V \in \mathcal{V}} \sum_{y \in Y_{V}}\left|O_{y} \cap f\left(X \cap U_{s}\right)\right| \leq \kappa$,

which is a desired contradiction proving that the set $f(X) \backslash Y_{s}$ is infinite.

Since $Y$ is functionally Hausdorff, we can choose two distinct points $y_{0}, y_{1} \in f(X) \backslash S$ and two open $F_{\sigma}$-sets $V_{0}, V_{1} \subset Y$ such that $\bar{V}_{0} \cap \bar{V}_{1}=\emptyset$ and $y_{i} \in V_{i}$ for $i \in\{0,1\}$. For every $i \in\{0,1\}$, the non-inclusion $y_{i} \notin Y_{s}$ ensures that $\left|V_{i} \cap f\left(X \cap U_{s}\right)\right|>\kappa$. It follows that $V_{s, i}:=U_{s} \cap f^{-1}\left(V_{i}\right)$ is an open $F_{\sigma}$-set in the open $F_{\sigma}$-subset $X \cap U_{s}$ of $X$. Consequently, $l\left(V_{s, i}\right) \leq l(X) \leq \kappa$. Find an open subset $W_{s, i} \subset U_{s} \cap W_{n}$ such that $V_{s, i}=X \cap W_{s, i}$. Consider the closure $\bar{W}_{s, i}$ of $W_{s, i}$ in $\bar{X}$ and observe that $f\left(X \cap \bar{W}_{s, i}\right) \subset \bar{V}_{i}$, which implies that $\bar{W}_{s, 0} \cap \bar{W}_{s, 1} \cap X=\emptyset$. Replacing the sets $W_{s, 0}$ and $W_{s, 1}$ by $W_{s, 0} \backslash \bar{W}_{s, 1}$ and $W_{s, 1} \backslash \bar{W}_{s, 0}$, respectively, we can assume that

$$
W_{s, 0} \cap \bar{W}_{s, 1}=\emptyset=W_{s, 1} \cap \bar{W}_{s, 0} .
$$

Since the space $\bar{X}$ is compact and Hausdorff, for every point $x \in V_{s, i}$ we can choose an open $\sigma$-compact neighborhood $O_{x}$ in $\bar{X}$ such that $\bar{O}_{x} \subset W_{s, i}$. Since $l\left(V_{s, i}\right) \leq \kappa$, there exists a set $X_{s, i} \subset V_{s, i}$ of cardinality $\left|X_{s, i}\right| \leq \kappa$ such that $V_{s, i} \subset \bigcup_{x \in X_{s, i}} O_{x}$ and hence $V_{s, i}=\bigcup_{x \in X_{s, i}} X \cap O_{x}$. Then $V_{i} \cap f\left(X \cap U_{s}\right)=f\left(V_{s, i}\right)=\bigcup_{x \in X_{s, i}} f\left(X \cap O_{x}\right)$. Since $\left|V_{i} \cap f\left(X \cap U_{s}\right)\right|>\kappa \geq\left|X_{s, i}\right|$, there exists a point $x_{s, i} \in X_{s, i}$ such that $\left|f\left(X \cap O_{x_{s, i}}\right)\right|>\kappa$. Put $U_{s^{i} i}:=O_{x_{s, i}}$, and observe that the sets $U_{s^{\wedge} 0}$ and $U_{s^{\wedge} 1}$ satisfy the conditions $\left(b_{s}\right),\left(c_{s}\right),\left(d_{s}\right)$ and $\left(a_{s^{\wedge} 0}\right),\left(a_{s^{\wedge} 1}\right)$.

After completing the inductive construction, consider the compact set $K=\bigcap_{n \in \omega} \bigcup_{s \in 2^{n}} \bar{U}_{s} \subset$ $\bigcap_{n \in \omega} W_{n+1}=X$. It remains to prove that the image $f(K) \subset Y$ is not scattered.

The conditions $\left(b_{s}\right),\left(c_{s}\right)$, of the inductive construction imply that for every point $x \in K$ there exists a unique sequence $s_{x} \in 2^{\omega}$ such that $x \in \bigcap_{n \in \omega} \bar{U}_{s_{x} \uparrow n}=\bigcap_{n \in \omega} U_{s\lceil n}$. It follows that the map $\varphi: K \rightarrow 2^{\omega}, \varphi: x \mapsto s_{x}$, is continuous and surjective. We claim that for any $x, y \in K$ 
with $\varphi(x) \neq \varphi(y)$ the points $f(x)$ and $f(y)$ are distinct. Since $s_{x}=\varphi(x) \neq \varphi(y)=s_{y}$, there exists a unique $n \in \omega$ such that $s_{x}\left\lceil n=s_{y}\left\lceil n\right.\right.$ but $s_{x}(n) \neq s_{y}(n)$. The inductive condition $\left(d_{t}\right)$ for $t=s_{x}\left\lceil n=s_{y}\left\lceil n\right.\right.$ ensures that $\{f(x)\} \cap\{f(y)\} \subset f\left(X \cap \bar{U}_{t^{\wedge} 0}\right) \cap f\left(X \cap \bar{U}_{t^{\wedge} 1}\right)=\emptyset$, which means that $f(x) \neq f(y)$. Then there exists a unique function $\phi: f(K) \rightarrow 2^{\omega}$ such that $\varphi=\phi \circ f\lceil K$. Taking into account that the map $\varphi$ is continuous and the map $f \nmid K: K \rightarrow f(K)$ is closed (and hence quotient), we conclude that the map $\phi: f(K) \rightarrow 2^{\omega}$ is continuous. So, $f(K)$ admits a continuous map onto $2^{\omega}$ and hence admits a surjective continuous map onto [0,1]. By Theorem 1 the compact space $f(K)$ is not scattered.

\section{AcKnowledgement}

The first author would like to express his thanks to Saak Gabriyelyan whose stimulating questions lead to the results presented in this paper. These results are exploited in the joint paper [4.

\section{REFERENCES}

[1] A.V. Arhangel'skii, Compactness, General Topology, II, 1-117, 247-256, Encyclopaedia Math. Sci., 50, Springer, Berlin, 1996.

[2] A.V. Arhangel'skii, Paracompactness, function spaces, descriptive theory, General Topology. III, Encyclopaedia Math. Sci., 51. Springer, Berlin, 1995. vi+229 pp.

[3] T. Banakh, K-analytic spaces whose any compact subset is countable, https://mathoverflow.net/q/328100/61536.

[4] T. Banakh, S. Gabriyelyan, Baire category properties of some Baire type function spaces, preprint.

[5] A. Blass, Combinatorial cardinal characteristics of the continuum, Handbook of Set Theory. Vols. 1, 2, 3, 395-489, Springer, Dordrecht, 2010.

[6] M.M. Choban, N.K. Dodon, Theory of P-scattered spaces (in Russian), Ştiinţa, Chişinău, 1979.

[7] E.K. van Douwen, The integers and topology, Handbook of Set-Theoretic Topology, 111-167, North-Holland, Amsterdam, 1984.

[8] R. Engelking, General Topology, Heldermann Verlag, Berlin, 1989.

[9] D.H. Fremlin, K-analytic spaces with metrizable compacta, Mathematika 24:2 (1977), 257-261.

[10] R. Hansell, Descriptive topology, Recent Progress in General Topology (Prague, 1991), 275-315, North-Holland, Amsterdam, 1992

[11] J. Kąkol, W. Kubiś, M. López-Pellicer, Descriptive Topology in Selected Topics of Functional Analysis, Springer, New York, 2011.

[12] A. Kechris, Classical Descriptive Set Theory, 156. Springer-Verlag, New York, 1995.

[13] C.A. Rogers, J.E. Jayne, K-analytic sets, in: Analytic Sets, Acad. Press, (1980), 1-181.

[14] Z. Semadeni, Banach Spaces of Continuous Functions, vol. 1, PWN - Polish Scientific Publishers, Warsaw 1971.

[15] V.V. Tkachuk, A $C_{p}$-Theory Problem Book. Special Features of Function Spaces, Springer, Cham, 2014.

[16] J. Vaughan, Small uncountable cardinals and topology, Open Problems in Topology, 195-216, North-Holland, Amsterdam, 1990.

T.Banakh: Jan Kochanowski University in Kielce (Poland) and Ivan Franko National University of LVIV (UKRAINE)

E-mail address: t.o.banakh@gmail.com

B.Bokalo: Ivan Franko National University of Lviv (Ukraine)

E-mail address: b.m.bokalo@gmail.com

V.Tkachuk: Universidad Autónoma Metropolitana (Mexico City, Mexico)

E-mail address: vova@xanum.uam.mx 